\title{
Quantitative Computerized Tomography (QCT) versus Dual X-Ray Absorptiometry (DXA) in the Assessment of Bone Mineral Density of HIV-1 Infected Children
}

\author{
Joann Lin ${ }^{1}$, Maria Ines Boechat ${ }^{2}$, Jaime G. Deville², Diego Gilsanz ${ }^{2}$, Richard Stiehm², \\ Vicente Gilsanz $^{3}$, Isidro Salusky ${ }^{2}$, Karin Nielsen-Saines ${ }^{2}$ \\ ${ }^{1}$ McKinney Allergy and Asthma Center, McKinney, USA; ${ }^{2}$ Department of Pediatrics, David Geffen UCLA School of Medicine, Los \\ Angeles, USA; ${ }^{3}$ Department of Radiology, Children's Hospital, Los Angeles, USA. \\ Email: knielsen@mednet.ucla.edu
}

Received August 29 $9^{\text {th }}, 2012$; revised September $26^{\text {th }}, 2012$; accepted October $10^{\text {th }}, 2012$

\begin{abstract}
Bone studies of HIV-infected children using dual X-ray absorptiometry (DXA) suggest bone mineral density (BMD) abnormalities. Pediatric studies are often performed using DXA instead of computed tomography (CT), which accounts for 3-dimensional differences in bone size of growing children. We evaluated whether CT would match DXA measurements in this population. For this purpose, the BMD of 16 perinatally HIV-infected patients, ages 6 to 22 was assessed. Subjects were matched by age, gender, and race to controls. BMD was assessed via DXA and QCT. Clinical anthropometric data, body mass index, immunologic and virologic parameters and laboratory markers for osteoblastic and osteoclastic activity were performed. No statistically significant differences in age and anthropometric parameters between subjects and controls were found. Individual CT and DXA z-scores were significantly different when subjects were evaluated as a group $(p=0.0002)$ or when males and females were analyzed independently $(p=0.001$ and 0.03$)$. DXA z-scores were below $1 \mathrm{SD}$, while $\mathrm{CT}$ z-scores were above the mean. $31 \%$ of subjects were identified as having poor bone mineralization by DXA while none had osteopenia/osteoporosis by CT. There was no correlation between immunologic/virologic parameters and BMD by either method. Increased osteoclastic activity was noted in 10 patients receiving tenofovir. In summary, decreased BMD diagnosed by DXA in pediatric HIV-infected subjects was not confirmed by CT. Increased bone turnover in patients on tenofovir was suggested by laboratory markers. Prospective studies using CT as the imaging standard are needed for evaluation of bone mineral changes in HIV-infected children.
\end{abstract}

Keywords: Bone Mineral Density; HIV-Infected Children; CT; DXA

\section{Introduction}

As children with HIV-infection survive into adolescence and adulthood, long term complications of their disease become apparent. In adults with prolonged HIV-infection and also prolonged antiretroviral treatment courses, metabolic disturbances such as losses in bone mineral density are not uncommon. Pathologic fractures can develop in HIV-infected patients who had no other risk factors for bone disease. Prolonged antiretroviral therapy, particularly protease inhibitors, and more recently developed nucleotide reverse transcriptase inhibitors (tenofovir) have been postulated to play a role in the rise in prevalence of osteopenia and osteoporosis in this patient population. Studies in children, however, have been few. Since $90 \%$ of the peak bone mass is achieved during childhood and adolescence, it is crucial to define the impact of HIV and highly active antiretroviral therapy
(HAART) on bone health and development in this age group.

Dual energy X-ray absorptiometry (DXA) is the most widely used technique for measuring bone acquisition in children because of its low cost, minimal radiation exposure, accessibility, and ease of use. Although the availability of DXA has resulted in many studies to diagnose and quantify the bone deficiency associated with pediatric disorders, it has become increasingly clear that major errors in interpretation occur when using this technique in children; errors which often may lead to the over-diagnosis of osteoporosis. Indeed, several investigators have proposed that osteoporosis should not be diagnosed based on DXA densitometry criteria alone $[1,2]$. A comparative study in 400 healthy children revealed that three times the number of subjects had bone values in the axial skeleton more than 2 standard deviations below the mean when using DXA as compared to 
computed tomography (CT) [3]. Studies suggest that DXA bone determinations frequently underestimate the amount of bone in children, regardless of age, sex, or whether they are healthy or sick.

Multiple studies in children and young adults have associated HIV infection with low bone mineral density (BMD) as measured via dual energy X-ray absorptiometry (DXA) as the main endpoint [4-10]. The etiology of BMD loss in pediatric HIV-infected patients is the subject of considerable debate. However, the imaging method employed to determine bone mineral loss may influence the findings. To determine whether the deficiency in bone mass previously reported in children with HIV-infection is in reality partly due to the imaging technique employed, we evaluated HIV pediatric patients for bone mineral density using both DXA and CT, a technique that provides bone measurements without the influence of surrounding soft tissues or bone size [11,12].

\section{Methods}

\subsection{Study Design}

A cross-sectional pilot study was conducted in a cohort of HIV-infected pediatric children and adolescents in Los Angeles.

\subsection{Population}

The study population was recruited from the Mattel Children's Hospital at the University of California Los Angeles Pediatric HIV Clinic from 2006 to 2009 during regular clinic visits. The Institutional Review Board approved the protocol and informed consent was obtained from all parents and/or subjects. Patients were physically active and all were receiving triple antiretroviral therapy. No patients were receiving other medications known to affect bone mineralization. Subjects with scoliosis of more than 20 degrees or kyphosis of more than 40 degrees, previous surgery with metal pins, rods, screws, or staples, a non-removable body piercing in the chest or abdomen, or current pregnancy were excluded. Study subjects were matched by race, gender, and age to a control group of healthy, uninfected children whose anthropometric measures and imaging findings by $\mathrm{CT}$ and DXA were recorded in an anonymous imaging database of national standards for healthy subjects generated in prior studies [13].

\subsection{Clinical Evaluations}

All patients underwent a complete physical examination. Weight was obtained to the nearest $0.1 \mathrm{~kg}$, using the Scale-Tronix (Scale-Tronix, Inc., Wheaton, Ill) and measurements of height and trunk height were obtained to the nearest $0.1 \mathrm{~cm}$, using the Harpenden stadiometer (Holtain Ltd., Crymmych, Wales). Body mass index (BMI) was calculated as weight $(\mathrm{kg})$ divided by height squared $\left(\mathrm{m}^{2}\right)$. Tanner stage of sexual development was assessed based on breast development in females and testicular size in males.

\subsection{Laboratory Assessments}

Bone-specific alkaline phosphate and osteocalcin, both markers of osteoblastic bone formation, were obtained at the UCLA General Clinical Research Center. Urinary N-terminal telopeptide of type I collagen (NTx) was measured as a marker of osteoclastic bone resorption. Plasma HIV RNA levels (Roche Amplicor Monitor 1.5; Roche Diagnostics, Alameda, CA) and T-cell subsets were performed. CDC clinical stage, CD4 cell counts, duration of antiretroviral therapy and HIV-1 viral load were recorded in order to assess HIV disease progression in these patients.

\subsection{CT and DXA Bone Measurements}

All CT studies were performed by the same radiology technologist with the same scanner (CT-T 9800; General Electric Co., Milwaukee, WI) and the same mineral reference phantom (CT-T bone densitometry package; General Electric Co., Milwaukee, WI). Identification of the sites to be scanned was performed with lateral scout views, and the density of cancellous bone in the lumbar vertebrae was obtained from the $10 \mathrm{~mm}$ midportion of the vertebral bodies. The average density was calculated and compared with published normative data from our laboratory [13] to determine the $\mathrm{CT}$ z-score. Coefficients of variation for repeated CT measurements of vertebral density were approximately $1.5 \%[13,14]$. The time required for the procedure was 10 minutes. The radiation dose ranged from 100 to $200 \mathrm{mrem}(1.5 \mathrm{mSv})$ localized to the midportions of the lumbar vertebrae; the effective radiation dose was approximately 8 mrem $[15,16]$.

DXA scanning was performed by the same radiology technologist using the same densitometer (Delphi W; Hologic, Inc., Waltham, MA). Anterior-posterior scans of the lumbar spine were obtained and bone mineral density (BMD) and z-scores were calculated according to the manufacturer's software. The time required for the procedure was approximately 5 minutes, and the radiation exposure was negligible $[17,18]$. Coefficients of variation for repeated DXA measurements of vertebral BMD and $\mathrm{BMC}$ reportedly range from $0.7 \%-1.7 \%$.

\subsection{Statistical Analysis}

Statistical analysis was carried out using Statview (version 5.01; SAS Institute Inc., Cary, NC). Antropometric 
and bone mineral density results were compared to that of healthy age, gender and ethnicity-matched HIV-uninfected controls. Linear regression was used to examine the relation between DXA versus CT bone measures and age, anthropometric, biochemical and immunological parameters. Paired t-test was used to compare DXA and CT z-scores.

\section{Results}

Sixteen subjects ( 11 males, 5 females) ages 6 to 22 years were enrolled in the study and completed all assessments. All 16 study subjects had anthropometric and imaging findings compared to that of 16 ages, gender and racematched uninfected controls. All 16 subjects had acquired HIV during the perinatal period; in 14 children, HIV infection was the result of vertical transmission from an HIV-infected mother, and two were infected by blood transfusions in infancy. The average age at diagnosis of HIV infection was 6 years $(5.97 \pm 5.2)$ and the average duration of triple antiretroviral treatment was 8 years $(7.8 \pm 2.1)$. On the day of the bone density determinations, 12 patients $(75 \%)$ were taking a protease inhibitor, and $10(63 \%)$ were receiving tenofovir disoproxil fumarate (DF). Disease severity was determined using CDC clinical stage; six patients were stage A, three were stage B and seven were stage C. Three patients (19\%) had CD4 counts less than 200/cmm, four (25\%) had CD4 counts $200-500 / \mathrm{cmm}$, and nine (56\%) had CD4 counts greater than $500 / \mathrm{cmm}$. Twelve patients $(75 \%)$ had HIV RNA copies below the dectectable cutoff of 50 copies $/ \mathrm{ml}$ of plasma. Demographic and clinical characteristics are shown in Table 1.

Three patients had elevated serum bone-specific alkaline phosphatase, and two had elevated serum osteocalcin levels. Ten patients had elevated urinary N-terminal telopeptide of type I collagen. All of the patients on tenofovir DF had elevated urinary NTx values.

Table 2 demonstrates the anthopometric characteristics of the study population and that of a control population used as normative data [13]. There were no statistically significant differences between both groups.

Age, anthropometric features, and CT and DXA bone measurements of the study subjects are described in Table 3. Male subjects tended to be underweight and shorter when compared to the appropriate controls. In contrast, the five female subjects had higher body mass indexes as compared to the respective controls. As seen in Figures 1 and 2, there were significant correlations between age, weight and height in male, (but not female) patients and DXA bone values; the strongest correlation occurred with weight. In contrast, regardless of gender, there were no correlations between age or anthropometric measures and $\mathrm{CT}$ values for bone density.
Table 1. Demographic and clinical characteristics.

\begin{tabular}{cc}
\hline Gender & 11 males $(79 \%)$ \\
\hline Race & 4 black $(36 \%)$ \\
& 7 hispanic $(44 \%)$ \\
& 5 white $(31 \%)$ \\
& $\mathrm{A}-6(38 \%)$ \\
CDC clinical stage & $\mathrm{B}-3(19 \%)$ \\
& $\mathrm{C}-7(44 \%)$ \\
CD4 count $(/ \mathrm{cmm})$ & $549 \pm 313$ \\
$\begin{array}{c}\text { Mean duration of } \\
\text { triple antiretroviral } \\
\text { regimens }\end{array}$ & $7.8 \pm 2.1 \mathrm{yrs}$ \\
\hline
\end{tabular}

Table 2. Demographic and anthropometric characteristics of study and control populations. Values described as mean and median range.

\begin{tabular}{ccc}
\hline & \multicolumn{2}{c}{ Subject parameters } \\
\cline { 2 - 3 } & HIV infected & Controls \\
\hline subjects & $14.2 \pm 4.4$ \\
Mean age (years) & $14.4 \pm 4.66$ & $13.7(6.1-22.8)$ \\
Median & $14(6-22.3)$ & 9.89 \\
Mean weight (kg) & 10.44 & $(4.91-17.3)$ \\
Median & $(4-19.7)$ & 0.815 \\
Mean height (cm) & 0.8275 & $(0.519-1.188)$ \\
Median & $(0.524-0.986)$ & -0.4 \\
Mean BMI & -1.05 & $(-2.5$ to 1.5$)$ \\
Median & $(-3.4$ to 0.7$)$ & 1847.7 \\
Mean Tanner & 1565.8 & $(712.5-1680.1)$ \\
Median & $(957-3224)$ &
\end{tabular}

When subjects were evaluated as a group, regardless of imaging technique, no association was observed between bone measurements and markers of bone formation or resorption, CD4 cell counts or percentages, or virus load as measured by HIV RNA PCR. All correlation coefficients ranged between -0.07 and 0.42 . There were, however, significant correlations between $\mathrm{CT}$ values for volumetric BD and DXA measures of areal BMD; this was true whether either absolute values or $\mathrm{z}$-scores were used in the comparisons, as illustrated in Figure 1.

Nevertheless, patient CT and DXA z-scores were significantly different. As shown in Figure 2, these differences were statistically significant when all subjects were evaluated as a group $(p=0.0002)$ and when males and females were analyzed independently $(p=0.001$ and 0.03 , respectively).

On average, DXA z-scores were just below $1 \mathrm{SD}$, while CT $\mathrm{z}$-scores were just above the mean $(-1.1 \pm 1.1$ and $0.3 \pm 1.4$ for DXA and CT, respectively). Five of 16 subjects $(31 \%)$ were identified as having poor bone mineralization by DXA. Three had bone densities lower than -1.5 SD (osteopenia), and an additional two had bone densities lower than $-2 \mathrm{SD}$ (osteoporosis). None were 
Table 3. DXA/QCT bone studies of HIV-1 infected subjects and healthy controls.

\begin{tabular}{|c|c|c|c|}
\hline \multirow{2}{*}{ DXA } & \multicolumn{3}{|c|}{ Subject parameters } \\
\hline & $\begin{array}{l}\text { HIV infected } \\
\text { subjects }\end{array}$ & Controls & $p$ \\
\hline Vertebral BMC (g) & $\begin{array}{c}10.44 \\
(4-19.7)\end{array}$ & $\begin{array}{c}10.44 \\
(4-19.7)\end{array}$ & 0.96 \\
\hline $\begin{array}{l}\text { Vertebral BMD } \\
\qquad\left(\mathrm{g} / \mathrm{cm}^{2}\right)\end{array}$ & $\begin{array}{c}0.8275 \\
(0.524-0.986)\end{array}$ & $\begin{array}{c}0.8275 \\
(0.524-0.986)\end{array}$ & 0.56 \\
\hline Vertebral z-score & $\begin{array}{c}-1.05 \\
(-3.4 \text { to } 0.7)\end{array}$ & $\begin{array}{c}-1.05 \\
(-3.4 \text { to } 0.7)\end{array}$ & 0.17 \\
\hline $\begin{array}{l}\text { Whole body BMC } \\
\text { (g) }\end{array}$ & $\begin{array}{c}1565.8 \\
(957-3224)\end{array}$ & $\begin{array}{c}1565.8 \\
(957-3224)\end{array}$ & 0.42 \\
\hline $\begin{array}{l}\text { Whole body area } \\
\qquad\left(\mathrm{cm}^{2}\right)\end{array}$ & $\begin{array}{c}1615.6 \\
(810-2051.6)\end{array}$ & $\begin{array}{c}1615.6 \\
(810-2051.6)\end{array}$ & 0.75 \\
\hline $\begin{array}{l}\text { Whole body BMD } \\
\left(\mathrm{g} / \mathrm{cm}^{2}\right)\end{array}$ & $\begin{array}{c}0.978 \\
(0.723-1.351) \\
\end{array}$ & $\begin{array}{c}0.9805 \\
(0.818-1.233) \\
\end{array}$ & 0.34 \\
\hline \multirow{2}{*}{ QCT } & \multicolumn{3}{|c|}{ Subject parameters } \\
\hline & $\begin{array}{l}\text { HIV infected } \\
\text { subjects }\end{array}$ & Controls & $p$ \\
\hline $\begin{array}{l}\text { Cancellous CSA } \\
\left(\mathrm{cm}^{2}\right)\end{array}$ & $\begin{array}{c}8.00 \\
(5.51-12.03)\end{array}$ & $\begin{array}{c}9.47 \\
(6.69-11.3)\end{array}$ & 0.15 \\
\hline $\begin{array}{l}\text { Cancellous BMD } \\
\left(\mathrm{mg} / \mathrm{cm}^{3}\right)\end{array}$ & $\begin{array}{c}272.5 \\
(243-360)\end{array}$ & $\begin{array}{c}293.4 \\
(237.8-498)\end{array}$ & 0.33 \\
\hline Cortical CSA $\left(\mathrm{cm}^{2}\right)$ & $\begin{array}{c}4.68 \\
(2.31-5.72)\end{array}$ & $\begin{array}{c}5.27 \\
(3.1-6.5)\end{array}$ & 0.51 \\
\hline $\begin{array}{l}\text { Cortical BMD } \\
\left(\mathrm{mg} / \mathrm{cm}^{3}\right)\end{array}$ & $\begin{array}{c}1178.9 \\
(387-1214.8)\end{array}$ & $\begin{array}{c}1220.3 \\
(1173-1295)\end{array}$ & 0.54 \\
\hline
\end{tabular}

BMD: Bone mineral density; BMC: Bone mineral concentration; CSA: Cross-sectional area.

shown to have osteopenia or osteoporosis by CT.

\section{Discussion}

The findings of our small pilot study corroborate previous pediatric studies which have indicated that HIV-infected children and adolescents have lower bone density values when assessed by DXA $[4,5,7,8]$. In contrast, when using CT to obtain measures of bone, we found no skeletal deficiencies in these patients. While z-scores for DXA were, on average, $1 \mathrm{SD}$ below reference values for healthy children, those for CT were close to the mean. Five children in the group were shown to have either osteopenia or osteoporosis, but findings were not reproducible with QCT. Although the number of patients evaluated was small, the high frequency of discordance between both imaging techniques supports the premise that reported bone deficiencies in DXA studies, particularly pediatric studies, are likely the result of technical conditions related to the technique as opposed to actual bone loss (as variations in soft tissue highly impact DXA measurements, particularly in children).

Interpretation of DXA bone studies is considerably
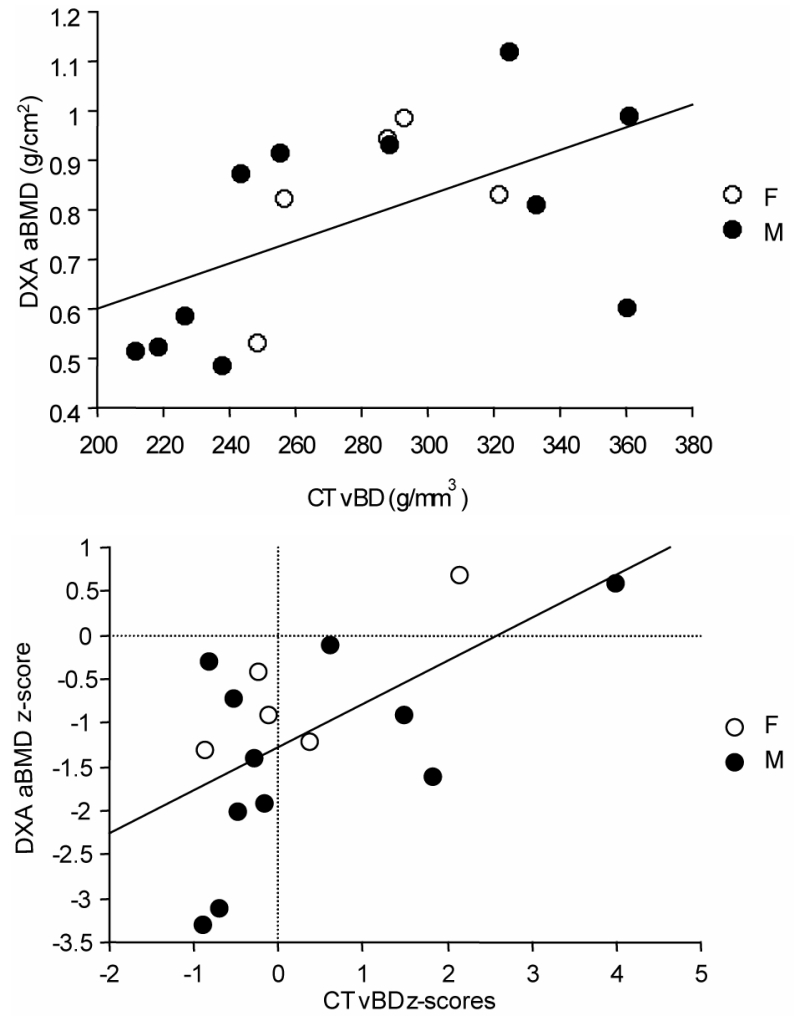

Figure 1. Correlations between absolute values and z-scores for CT BD values and DXA BMD.

more challenging in children than in adults and several investigators have found that osteoporosis in children is frequently misdiagnosed secondary to results from DXA scans $[2,19,20]$. Because DXA measurements are known to be affected by the composition of soft tissue surrounding the targeted bone, values are based on the assumption that the proportions of lean and fat tissues around the bone are constant and similar in all subjects. Unfortunately, the inability of DXA determinations to account for inhomogeneous growth-related variations in body composition especially limits comparative studies on the effects of HIV-infection on the skeleton. In the current study, measures of CT bone density, in contrast to those of DXA, were not related to anthropometric parameters, disputing previous suggestions that low body weight mediates the relation between HIV and low BMD and that differences in body composition account for the high prevalence of low BMD in these patients.

The cross-sectional examination of only the axial skeleton in a relatively small number of subjects with a narrow age range are limitations of this study. However, the use of two imaging modalities to assess bone density and the use of the same technologist to obtain all CT and DXA measurements are major strengths. Nevertheless, even with this small sample population, significant differences between CT and DXA values in HIV-infected 


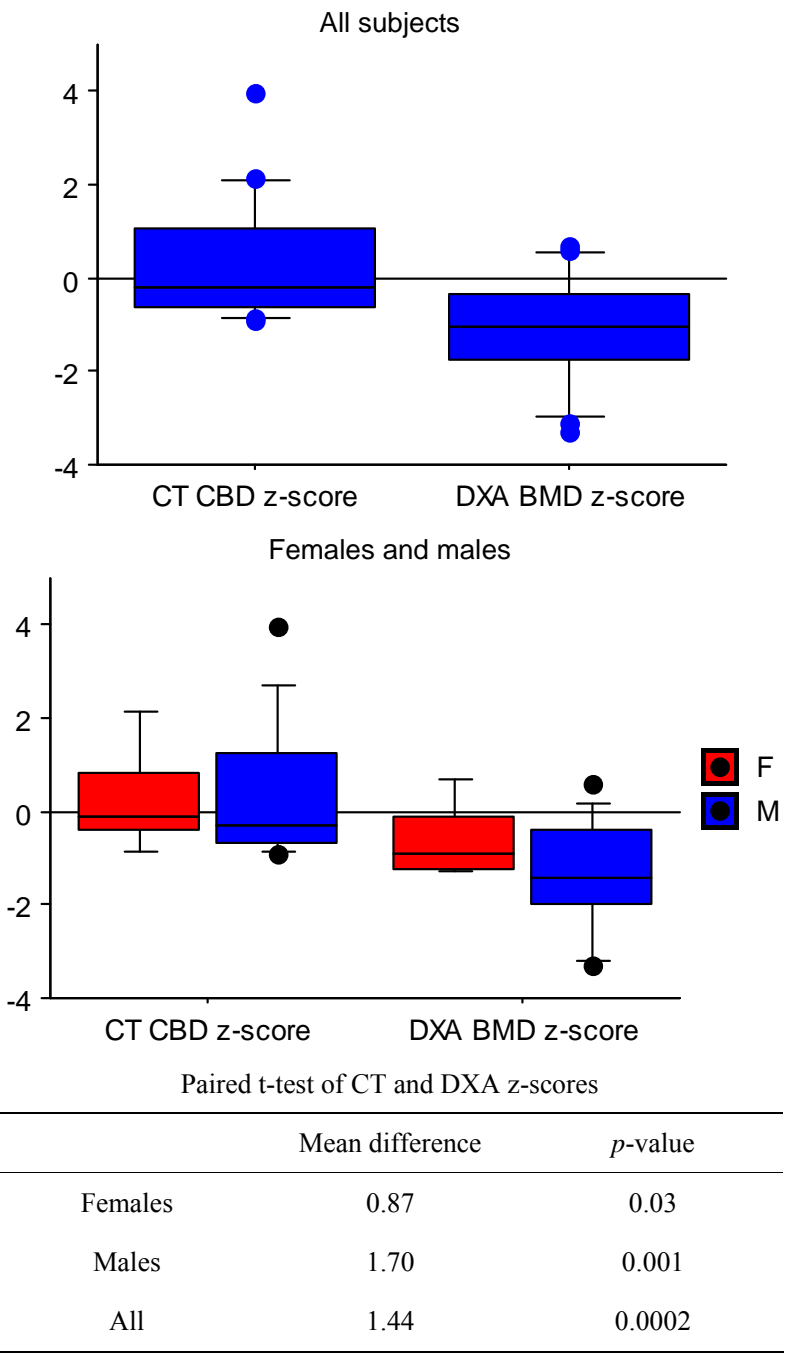

Figure 2. Box and whisker plots of CT BD z-scores and DXA BMD z-scores in all subjects in all subjects, and in females and males, independently, showing highly significant differences between techniques.

patients were revealed. Future studies are needed to establish whether these results also hold for older populations and other skeletal sites or if prospective follow-up provides more consistent and reliable parameters in this population as it may in other groups [21]. Our findings are relevant, however, as ease of use and negligible amounts of radiation exposure make DXA imaging an attractive resource for evaluation of bone density. As concerns for potential bone mineralization loss in antiretroviral-treated children increase and bone assessments become part of routine care, it is critical that providers become aware of the inherent variability of this technique, when compared to other imaging modalities, particularly in growing children, as the measure is highly discrepant to the gold standard (CT) and tends to overread bone loss.
The laboratory finding of increased osteoclastic activity in all patients receiving tenofovir diproxil fumarate requires further investigation. Although radiologic evidence of BMD did not correlate with laboratory findings, patients may not have had sufficient time of tenofovir exposure for BMD loss to become apparent.

In summary, results of the present study support the notion that diagnosis of osteoporosis or decisions to treat low bone mass in HIV-infected children should be made using strict criteria and should not be based solely on DXA findings. Our results suggest that it would not be appropriate for HIV-infected children on antiretroviral therapy to routinely be monitored though bone densitometry.

\section{Acknowledgements}

This research was funded by The Elizabeth Glaser Pediatric Research Foundation through a fellowship award for Joann Lin, and the UCLA General Clinical Research Center M01-RR00865 which provided support for the laboratory studies. The authors do not have any conflicts of interest to disclose.

\section{REFERENCES}

[1] The Writing Group for the ICSD Position Development Conference, "Diagnosis of Osteoporosis in Men, Premenopausal Women, and Children," Journal of Clinical Densitometry, Vol. 7, No. 1, 2004, pp. 17-26.

[2] R. I. Gafni and J. Baron, "Overdiagnosis of Osteoporosis in Children Due to Misinterpretation of Dual-Energy X-Ray Absorptiometry (DEXA)," Journal of Pediatrics, Vol. 144, No. 2, 2004, pp. 253-257. doi:10.1016/j.jpeds.2003.08.054

[3] T. A. L. Wren, X. Liu, P. Pitukcheewanont and V. Gilsanz, "Bone Densitometry in Pediatric Populations: Discrepancies in the Diagnosis of Osteoporosis by DXA and CT," Journal of Pediatrics, Vol. 146, No. 6, 2005, pp. 776-779. doi:10.1016/j.jpeds.2005.01.028

[4] S. M. Arpadi, M. Horlick, J. Thornton, P. A. Cuff, J. Wang and D. P. Kotler, "Bone Mineral Content Is Lower in Prepubertal HIV-Infected Children," Journal of Acquired Immune Deficiency Syndrome, Vol. 19, 2002, pp. 450-454.

[5] M. J. Bolland, A. B. Grey, G. D. Gamble and I. R. Reid, "Low Body Weight Mediates the Relationship between HIV-Infection and Low Bone Mineral Density: A MetaAnalysis," Journal of Clinical Endocrinology and Metabolism, Vol. 92, No. 12, 2007, pp. 4522-4528. doi: $10.1210 /$ jc. $2007-1660$

[6] J. H. Arnsten, R. Freeman, A. A. Howard, M. FlorisMoore, Y. Lo and R. S. Klein, "Decreased Bone Mineral Density and Increased Fracture Risk in Aging Men with or at Risk for HIV Infection," AIDS, Vol. 21, No. 5, 2007, pp. 617-623. doi:10.1097/QAD.0b013e3280148c05 
[7] J. B. Purdy, R. I. Gafni, J. C. Reynolds, S. Zeichner and R. Hazra, "Decreased Bone Mineral Density with Off-Label Use of Tenofovir in Children and Adolescents Infected with Human Immunodeficiency Virus," Journal of Pediatrics, Vol. 152, No. 4, 2008, pp. 582-584. doi:10.1016/j.jpeds.2007.12.020

[8] S. Mora, I. Zamproni, S. Beccio, R. Bianchi, V. Giacomet and A. Vigano, "Longitudinal Changes of Bone Mineral Density and Metabolism in Antiretroviral-Treated Human Immunodeficiency Virus-Infected Children," Journal of Clinical Endocrinology \& Metabolism, Vol. 89, No. 1, 2004, pp. 24-28. doi:10.1210/jc.2003-030767

[9] S. Landonio, T. Quirino, P. Bonfanti, et al., "Osteopenia and Osteoporosis in HIV+ Patients, Untreated or Receiving HAART," Biomedical Pharmacotherapy, Vol. 58, No. 9, 2004, pp. 505-508. doi:10.1016/j.biopha.2004.08.019

[10] S. E. Dolan, J. S. Huang, K. M. Killilea, M. P. Sullivan, N. Aliabadi and S. Grinspoon, "Reduced Bone Density in HIV-Infected Women," AIDS, Vol. 18, No. 3, 2004, pp. 475-483. doi:10.1097/00002030-200402200-00014

[11] V. Gilsanz, "Bone Density in Children: A Review of the Available Techniques and Indications," European Journal of Radiology, Vol. 26, No. 2, 1998, pp. 177-182. doi:10.1016/S0720-048X(97)00093-4

[12] T. N. Hangartner and C. C. Johnston, "Influence of Fat on Bone Measurements with Dual-Energy Absorptiometry," Bone and Mineral, Vol. 9, No. 1, 1990, pp. 71-81. doi:10.1016/0169-6009(90)90101-K

[13] V. Gilsanz, F. J. Perez, P. P. Campbell, F. J. Dorey, D. C. Lee and T. A. Wren, "Quantitative CT Reference Values for Vertebral Trabecular Bone Density in Children and Young Adults," Radiology, Vol. 250, 2009, pp. 222-227. doi:10.1148/radiol.2493080206

[14] T. Hangartner and V. Gilsanz, "Evaluation of Cortical
Bone by Computed Tomography," Journal of Bone Mineralization Research, Vol. 11, 1996, pp. 1518-1525.

[15] V. Gilsanz, M. I. Boechat, T. F. Roe, M. L. Loro, J. W. Sayre and W. G. Goodman, "Gender Differences in Vertebral Body Sizes in Children and Adolescents," Radiology, Vol. 190, No. 3, 1994, pp. 673-677.

[16] W. Kalender, "Effective Dose Values in Bone Mineral Measurements by Photon Absorptiometry and Computed Tomography," Osteoporosis International, Vol. 2, No. 2, 1992, pp. 82-87. doi:10.1007/BF01623841

[17] C. E. Cann, "Why, When and How to Measure Bone Mass: A Guide for the Beginning User," In: G. D. Frey, M. V. Yester, Eds., Expanding the Role of Medical Physics in Nuclear Medicine, American Institute of Physics Publishing, New York, 1991, pp. 250-285.

[18] S. Mora, L. Bachrach and V. Gilsanz, "Noninvasive Techniques for Bone Mass Measurement," San Diego Academic Press, San Diego, 2003.

[19] M. B. Leonard, K. J. Propert, B. S. Zemel, V. A. Stallings and H. I. Feldman, "Discrepancies in Pediatric Bone Mineral Density Reference Data: Potential for Misdiagnosis of Osteopenia," Journal of Pediatrics, Vol. 135, No. 2, 1999, pp. 182-188. doi:10.1016/S0022-3476(99)70020-X

[20] A. Prentice, T. J. Parsons and T. J. Cole, "Uncritical Use of Bone Mineral Density in Absorptiometry May Lead to Size-Related Artifacts in the Identification of Bone Mineral Determinants," American Journal of Clinical Nutrition, Vol. 60, No. 6, 1994, pp. 837-842.

[21] H. J. Kalkwarf, V. Gilsanz, J. M. Lappe, et al., "Tracking of Bone Mass and Density during Childhood and Adolescence," Journal of Clinical Endocrinology and Metabolism, Vol. 95, No. 4, 2010, pp. 1690-1698. doi:10.1210/jc.2009-2319 